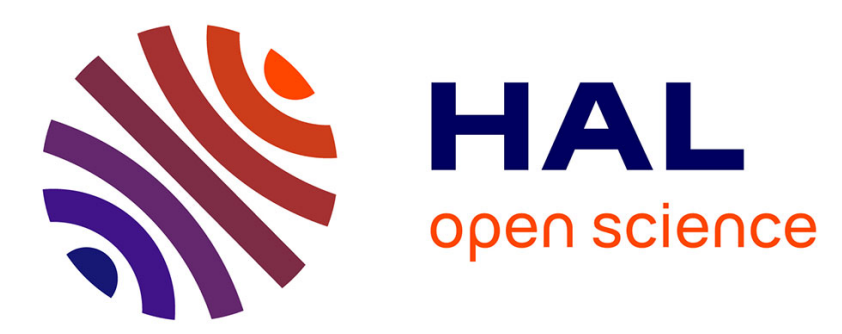

\title{
Optimal Control versus Stochastic Target problems: An Equivalence Result
}

Bruno Bouchard, Minh Ngoc Dang

\section{To cite this version:}

Bruno Bouchard, Minh Ngoc Dang. Optimal Control versus Stochastic Target problems: An Equivalence Result. Systems and Control Letters, 2012, 61 (2), pp.343-346. 10.1016/j.sysconle.2011.11.010 . hal-00556509

\section{HAL Id: hal-00556509 \\ https://hal.science/hal-00556509}

Submitted on 17 Jan 2011

HAL is a multi-disciplinary open access archive for the deposit and dissemination of scientific research documents, whether they are published or not. The documents may come from teaching and research institutions in France or abroad, or from public or private research centers.
L'archive ouverte pluridisciplinaire HAL, est destinée au dépôt et à la diffusion de documents scientifiques de niveau recherche, publiés ou non, émanant des établissements d'enseignement et de recherche français ou étrangers, des laboratoires publics ou privés. 


\title{
Optimal Control versus Stochastic Target problems: An Equivalence Result
}

\author{
Bruno Bouchard \\ CEREMADE, Université Paris Dauphine \\ and CREST-ENSAE \\ bouchard@ceremade.dauphine.fr
}

\author{
Ngoc Minh Dang \\ CEREMADE, Université Paris Dauphine \\ and CA Cheuvreux \\ dang@ceremade.dauphine.fr
}

January 17, 2011

\begin{abstract}
Within a general abstract framework, we show that any optimal control problem in standard form can be translated into a stochastic target problem as defined in [17], whenever the underlying filtered probability space admits a suitable martingale representation property. This provides a unified way of treating these two classes of stochastic control problems. As an illustration, we show, within a jump diffusion framework, how the Hamilton-Jacobi-Bellman equations associated to an optimal control problem in standard form can be easily retrieved from the partial differential equations associated to its stochastic target counterpart.
\end{abstract}

Key words: Stochastic target, stochastic control, viscosity solutions.

Mathematical subject classifications: Primary 49-00; secondary 49L25.

\section{Introduction}

In their simplest form, stochastic target problems can be formulated as follows. Given a controlled process $Z_{t, x, y}^{\nu}=\left(X_{t, x}^{\nu}, Y_{t, x, y}^{\nu}\right)$, associated to the initial condition $Z_{t, x, y}^{\nu}(t)=(x, y) \in$ $\mathbb{R}^{d} \times \mathbb{R}$ at time $t$, find the set $S(t)$ of initial conditions $(x, y)$ such that $\Psi\left(X_{t, x}^{\nu}(T), Y_{t, x, y}^{\nu}(T)\right) \geq 0$ $\mathbb{P}$ - a.s. for some control $\nu \in \mathcal{U}$, where $\Psi$ is a given real valued Borel measurable map and $\mathcal{U}$ is a prescribed set of controls.

When $y \mapsto Y_{t, x, y}^{\nu}(T)$ and $y \mapsto \Psi(\cdot, y)$ are non-decreasing, for all $\nu \in \mathcal{U}$, then the set $S(t)$ can be identified to $\left\{(x, y) \in \mathbb{R}^{d} \times \mathbb{R}: y \geq v(t, x)\right\}$ where $v(t, x):=\inf \{y \in \mathbb{R}: \exists \nu \in \mathcal{U}$ s.t. $\Psi\left(Z_{t, x, y}^{\nu}(T)\right) \geq 0 \mathbb{P}$ - a.s. $\}$, whenever the above infimum is achieved.

Such problems can be viewed as a natural generalization of the so-called super-hedging problem in mathematical finance. In this case, $Y_{t, x, y}^{\nu}$ is interpreted as the wealth process associated to a given investment policy $\nu, X_{t, x}^{\nu}$ as stock prices or factors (that can possibly be influenced by the trading strategy) and $\Psi$ takes the form $\Psi(x, y)=y-g(x)$ where $g$ is viewed as the payoff function of an European option. Then, $\Psi\left(X_{t, x}^{\nu}(T), Y_{t, x, y}^{\nu}(T)\right) \geq 0$ means $Y_{t, x, y}^{\nu}(T) \geq g\left(X_{t, x}^{\nu}(T)\right)$, i.e. the value of the hedging portfolio is greater at time $T$ than the payoff $g\left(X_{t, x}^{\nu}(T)\right)$ of the European claim. The value function $v(t, x)$ then coincides with the super-hedging price of the option, see e.g. [13] for references on mathematical finance.

Motivated by the study of super-hedging problems under Gamma constraints, Soner and Touzi [15] were the first to propose a direct treatment of a particular class of stochastic target 
problems. It relies on a Geometric Dynamic Programming Principle (GDP) which essentially asserts that $S(t)=\left\{(x, y) \in \mathbb{R}^{d} \times \mathbb{R}: \exists \nu \in \mathcal{U}\right.$ s.t. $Z_{t, x, y}^{\nu}(\theta) \in S(\theta)$ a.s. $\}$ for all $[t, T]$-valued stopping time $\theta$. The main observation of Soner and Touzi is that it actually allows one to provide a direct characterization of the associated value function $v$ as a viscosity solution of a non-linear parabolic partial differential equation. This approach was further exploited in [6] and [18], in the context of super-hedging problems in mathematical finance. A general version of the GDP was then proved in [17], where the authors also used this methodology to provide a new probabilistic representation of a class of geometric flows. The link with PDEs in a general Markovian framework for Brownian diffusion processes was established in [16], and extended to jump diffusion processes in [1] whose main motivation was to apply this approach to provision management in insurance. Finally, an extension to path dependent constraints was proposed in [8].

This approach turned out to be very powerful to study a large family of non-standard stochastic control problems in which a target has to be reached with probability one at a given time horizon $T$. However, it was limited to this case, up to the paper [5] who showed how the a.s. constraint $\Psi\left(Z_{t, x, y}^{\nu}(T)\right) \geq 0$ can indeed be relaxed in moment constraints of the form $\mathbb{E}\left[\Psi\left(Z_{t, x, y}^{\nu}(T)\right)\right] \geq p$, where the real number $p$ is a given threshold, typically non-positive. This relaxed version was called stochastic target problem with controlled loss in [5].

The result of [5] (extended by [14] to jump diffusion processes) opened the door to a wide range of new applications. In particular in mathematical finance, in which a $\mathbb{P}$-a.s. constraint would typically lead to degenerate results, i.e. $v \equiv \infty$ or $v$ much too large in comparison to what can be observed in practice, while the above relaxation provides meaningful results. A good illustration of such a situation in given in [3] which discusses the pricing of financial book liquidation contracts. See also the forthcoming paper [9] on the problem of P\&L matching in option hedging or optimal investment problems.

In view of all the potential practical applications of the technology originally proposed by Soner and Touzi in [15], and given the fact that the theory is now well-established, it seems natural to consider this recently developed class of (non-standard) stochastic control problems as a part of the general well-known tool box in optimal control. However, it seems a-priori that stochastic target problems and optimal control problems in standard form (i.e. expected cost minimization problems) have to be discussed separately as they rely on different dynamic programming principles.

This short note can be viewed as a teacher's note that explains why they can actually be treated in a unified framework. More precisely: any optimal control problem in standard form admits a (simple and natural) representation in terms of a stochastic target problem.

In the following, we first discuss this equivalence result in a rather general abstract framework. In the next section, we show through a simple example how the Hamilton-Jacobi-Bellman equations of an optimal control problem in standard form can be easily recovered from the PDEs associated to the corresponding stochastic target problem.

We will not provide in this short note the proof of the PDE characterization for stochastic target problems. We refer to [5] and [14] for the complete arguments.

Notations: We denote by $x^{i}$ the $i$-th component of a vector $x \in \mathbb{R}^{d}$, which will always be viewed as a column vector, with transposed vector $x^{\top}$, and Euclidean norm $|x|$. The set $\mathbb{M}^{d}$ is the collection of $d$-dimensional square matrices $A$ with coordinates $A^{i j}$, and norm $|A|$ defined by viewing $A$ as an element of $\mathbb{R}^{d \times d}$. Given a smooth function $\varphi:(t, x) \in \mathbb{R}_{+} \times \mathbb{R}^{d} \rightarrow \mathbb{R}$, we denote by $\partial_{t} \varphi$ its derivative with respect to its first variable, we write $D \varphi$ and $D^{2} \varphi$ for the Jacobian and Hessian matrix with respect to $x$. The set of continuous function $C^{0}(B)$ on a 
Borel subset $B \subset \mathbb{R}_{+} \times \mathbb{R}^{d}$ is endowed with the topology of uniform convergence on compact sets. Any inequality or inclusion involving random variables has to be taken in the a.s. sense.

\section{The equivalence result}

Let $T$ be a finite time horizon, given a general probability space $(\Omega, \mathcal{F}, \mathbb{P})$ endowed with a filtration $\mathbb{F}=\left\{\mathcal{F}_{t}\right\}_{t \leq T}$ satisfying the usual conditions. We assume that $\mathcal{F}_{0}$ is trivial.

Let us consider an optimal control problem defined as follows. First, given a set $U$ of deterministic functions from $\mathbb{R}_{+}$to $\mathbb{R}^{\kappa}, \kappa \geq 1$, we define

$$
\tilde{\mathcal{U}}=\{\nu: \mathbb{F} \text {-predictable process s.t. } t \longmapsto \nu(t, \omega) \in U \text { for } \mathbb{P} \text {-almost every } \omega \in \Omega\} .
$$

The controlled terminal cost is a map

$$
\nu \in \tilde{\mathcal{U}} \longmapsto G^{\nu} \in L^{0}\left(\Omega, \mathcal{F}_{T}, \mathbb{P}\right) .
$$

Without loss of generality, we can restrict to a subset of controls $\mathcal{U} \subset \tilde{\mathcal{U}}$ such that

$$
\mathcal{U} \subset\left\{\nu \in \tilde{\mathcal{U}}: G^{\nu} \in L^{1}\left(\Omega, \mathcal{F}_{T}, \mathbb{P}\right)\right\} .
$$

Given $(t, \nu) \in[0, T] \times \mathcal{U}$, we can then define the conditional optimal expected cost:

$$
V_{t}^{\nu}:=\operatorname{ess} \inf _{\mu \in \mathcal{U}(t, \nu)} \mathbb{E}\left[G^{\mu} \mid \mathcal{F}_{t}\right]
$$

where

$$
\mathcal{U}(t, \nu):=\{\mu \in \mathcal{U}: \mu=\nu \text { on }[0, t] \mathbb{P}-\text { a.s. }\} .
$$

Our main observation is that the optimal control problem (2.1) can be interpreted as a stochastic target problem involving an additional controlled process chosen in a suitable family of martingales. Moreover, existence in one problem is equivalent to existence in the other.

Lemma 2.1. [Stochastic target representation] Let $\mathcal{M}$ be a any family of martingales such that

$$
\mathcal{G}:=\left\{G^{\nu}, \nu \in \mathcal{U}\right\} \subset\left\{M_{T}, M \in \mathcal{M}\right\} .
$$

Then, for each $(t, \nu) \in[0, T] \times \mathcal{U}$ :

$$
V_{t}^{\nu}=Y_{t}^{\nu}
$$

where

$$
Y_{t}^{\nu}:=\operatorname{essinf}\left\{Y \in L^{1}\left(\Omega, \mathcal{F}_{t}, \mathbb{P}\right) \mid \exists(M, \mu) \in \mathcal{M} \times \mathcal{U}(t, \nu) \text { s.t. } Y+M_{T}-M_{t} \geq G^{\mu}\right\} .
$$

Moreover, there exists $\hat{\mu} \in \mathcal{U}(t, \nu)$ such that

$$
V_{t}^{\nu}=\mathbb{E}\left[G^{\hat{\mu}} \mid \mathcal{F}_{t}\right]
$$

if and only if there exists $(\bar{M}, \bar{\mu}) \in \mathcal{M} \times \mathcal{U}(t, \nu)$ such that

$$
Y_{t}^{\nu}+\bar{M}_{T}-\bar{M}_{t} \geq G^{\bar{\mu}} .
$$

In this case, one can choose $\bar{\mu}=\hat{\mu}$, and $\bar{M}$ satisfies

$$
Y_{t}^{\nu}+\bar{M}_{T}-\bar{M}_{t}=G^{\bar{\mu}} .
$$


Let us make some remarks before to provide the short proof.

Remark 2.2. It is clear that a family $\mathcal{M}$ satisfying (2.2) always exists. In particular, one can take $\mathcal{M}=\overline{\mathcal{M}}:=\left\{\left(\mathbb{E}\left[G^{\nu} \mid \mathcal{F}_{t}\right]\right)_{t \geq 0}, \quad \nu \in \mathcal{U}\right\}$. When the filtration is generated by a $d$ dimensional Brownian motion $W$, then the martingale representation theorem allows one to rewrite any element $M$ of $\overline{\mathcal{M}}$ in the form $M=P_{0, M_{0}}^{\alpha}:=M_{0}+\int_{0}^{\cdot} \alpha_{s}^{\top} d W_{s}$ where $\alpha$ belongs to $\mathcal{A}_{\text {loc }}$, the set of $\mathbb{R}^{d}$-valued locally square integrable predictable processes such that $P_{0,0}^{\alpha}$ is a martingale. This allows choosing $\mathcal{M}$ as $\left\{P_{0, p}^{\alpha},(p, \alpha) \in \mathbb{R} \times \mathcal{A}_{\text {loc }}\right\}$. When $\mathcal{G} \subset L^{2}\left(\Omega, \mathcal{F}_{T}, \mathbb{P}\right)$, then we can replace $\mathcal{A}_{\text {loc }}$ by the set $\mathcal{A}$ of $\mathbb{R}^{d}$-valued square integrable predictable processes. A similar reduction can be obtained when the filtration is generated by Lévy processes. We shall see below in Section 3 that such classes of families $\mathcal{M}$ allow us to convert a Markovian optimal control problem in standard form into a Markovian stochastic target problem for which a PDE characterization can be derived. A similar idea was already used in [5] to convert stochastic target problems under controlled loss, i.e. with a constraint in expectation, into regular stochastic target problems, i.e. associated to a $\mathbb{P}-$ a.s.-constraint, see their Section 3.

Remark 2.3. A similar representation result was obtained in [2] where it is shown that a certain class of optimal switching problems can be translated into stochastic target problems for jump diffusion processes. In this paper, the author also introduces an additional controlled martingale part but the identification of the two control problems is made through their associated PDEs (and a suitable comparison theorem) and not by pure probabilistic arguments. Moreover, an additional randomization of the switching policy is introduced in order to remove this initial control process.

Remark 2.4. It is well-known that, under mild assumptions (see [11]), the map $t \in[0, T] \mapsto$ $V_{t}^{\nu}$ can be aggregated by a càdlàd process $\bar{V}^{\nu}$ which is a submartingale for each $\nu \in \mathcal{U}$, and that a control $\hat{\mu}$ is optimal for (2.1) if and only if $\bar{V}^{\hat{\mu}}$ is a martingale on $[t, T]$. This is indeed a consequence of the dynamic programming principle which can be (at least formally) stated as $V_{t}^{\nu}=\operatorname{essinf}\left\{\mathbb{E}\left[V_{\theta}^{\mu} \mid \mathcal{F}_{t}\right], \mu \in \mathcal{U}(t, \nu)\right\}$ for all stopping time $\theta$ with values in $[t, T]$. It is clear from the proof below that the additional controlled process $\left(Y_{t}^{\nu}+\bar{M}_{s}-\bar{M}_{t}\right)_{s \geq t}$ whose $T$-value appears in (2.7) then coincides with this martingale.

Proof of Lemma 2.1 a. We first prove (2.3). To see that $Y_{t}^{\nu} \geq V_{t}^{\nu}$, fix $Y \in L^{1}\left(\Omega, \mathcal{F}_{t}, \mathbb{P}\right)$ and $(M, \mu) \in \mathcal{M} \times \mathcal{U}(t, \nu)$ such that $Y+M_{T}-M_{t} \geq G^{\mu}$. Then, by taking the conditional expectation on both sides, we obtain $Y \geq \mathbb{E}\left[G^{\mu} \mid \mathcal{F}_{t}\right] \geq \operatorname{essinf}\left\{\mathbb{E}\left[G^{\mu^{\prime}} \mid \mathcal{F}_{t}\right], \mu^{\prime} \in \mathcal{U}(t, \nu)\right\}$ $=V_{t}^{\nu}$, which implies that $Y_{t}^{\nu} \geq V_{t}^{\nu}$.

On the other hand, (2.2) implies that, for each $\mu \in \mathcal{U}(t, \nu)$, there exists $M^{\mu} \in \mathcal{M}$ such that $G^{\mu}=\mathbb{E}\left[G^{\mu} \mid \mathcal{F}_{t}\right]+M_{T}^{\mu}-M_{t}^{\mu}$. In particular, $\mathbb{E}\left[G^{\mu} \mid \mathcal{F}_{t}\right]+M_{T}^{\mu}-M_{t}^{\mu} \geq G^{\mu}$. This shows that $\mathbb{E}\left[G^{\mu} \mid \mathcal{F}_{t}\right] \geq Y_{t}^{\nu}$ for all $\mu \in \mathcal{U}(t, \nu)$, and therefore $V_{t}^{\nu} \geq Y_{t}^{\nu}$.

b. We now assume that $\hat{\mu} \in \mathcal{U}(t, \nu)$ is such that (2.5) holds. Since $V_{t}^{\nu}=Y_{t}^{\nu}$, this leads to $Y_{t}^{\nu}=\mathbb{E}\left[G^{\hat{\mu}} \mid \mathcal{F}_{t}\right]$. Moreover, $(2.2)$ implies that there exists $\bar{M} \in \mathcal{M}$ such that $\mathbb{E}\left[G^{\hat{\mu}} \mid \mathcal{F}_{t}\right]+$ $\bar{M}_{T}-\bar{M}_{t}=G^{\hat{\mu}}$. Hence, (2.6) and (2.7) hold with $\bar{\mu}=\hat{\mu}$. Conversely, if $(\bar{M}, \bar{\mu}) \in \mathcal{M} \times \mathcal{U}(t, \nu)$ is such that (2.6) holds, then the identity (2.3) implies that $V_{t}^{\nu}=Y_{t}^{\nu} \geq \mathbb{E}\left[G^{\bar{\mu}} \mid \mathcal{F}_{t}\right] \geq V_{t}^{\nu}$. Hence, (2.5) holds with $\hat{\mu}=\bar{\mu}$.

\section{Example}

In this section, we show how the Hamilton-Jacobi-Bellman equations associated to an optimal control problem in standard form can be deduced from its stochastic target formulation. We 
restrict to a classical case where the filtration is generated by a $d$-dimensional Brownian motion $W$ and a $E$-marked integer-valued right-continuous point process $N(d e, d t)$ with predictable $(\mathbb{P}, \mathbb{F})$-intensity kernel $m(d e) d t$ such that $m(E)<\infty$ and $\operatorname{supp}(m)=E$, where supp denotes the support and $E$ is a Borel subset of $\mathbb{R}^{d}$ with Borel tribe $\mathcal{E}$. We denote by $\tilde{N}(d e, d t)=$ $N(d e, d t)-m(d e) d t$ the associated compensated random measure, see e.g. [10] for details on random jump measures.

The set of controls $\mathcal{U}$ is now defined as the collection of square integrable predictable $K$-valued processes, for some $K \subset \mathbb{R}^{d}$. Given $\nu \in \mathcal{U}$ and $(t, x) \in[0, T] \times \mathbb{R}^{d}$, we define $X_{t, x}^{\nu}$ as the unique strong solution on $[t, T]$ of

$$
X^{\nu}=x+\int_{t} \mu\left(X^{\nu}(r), \nu_{r}\right) d r+\int_{t}^{\cdot} \sigma\left(X^{\nu}(r), \nu_{r}\right) d W_{r}+\int_{t} \int_{E} \beta\left(X^{\nu}(r-), \nu_{r}, e\right) N(d e, d r),(3
$$

where $(\mu, \sigma, \beta):(x, u, e) \in \mathbb{R}^{d} \times K \times E \longmapsto \mathbb{R}^{d} \times \mathbb{M}^{d} \times \mathbb{R}^{d}$ are measurable and are assumed to be such that there exists $L>0$ for which

$$
\begin{gathered}
\left|\mu(x, u)-\mu\left(x^{\prime}, u\right)\right|+\left|\sigma(x, u)-\sigma\left(x^{\prime}, u\right)\right|+\left(\int_{E}\left|\beta(x, u, e)-\beta\left(x^{\prime}, u, e\right)\right|^{2} m(d e)\right)^{\frac{1}{2}} \leq L\left|x-x^{\prime}\right| \\
|\mu(x, u)|+|\sigma(x, u)|+\underset{e^{\prime} \in E}{\operatorname{esssup}}\left|\beta\left(x, u, e^{\prime}\right)\right| \leq L(1+|x|+|u|),
\end{gathered}
$$

for all $x, x^{\prime} \in \mathbb{R}^{d}$ and $u \in K$.

Given a continuous map $g$ with linear growth (for sake of simplicity), we then define the optimal control problem in standard form:

$$
v(t, x):=\inf _{\nu \in \mathcal{U}} \mathbb{E}\left[g\left(X_{t, x}^{\nu}(T)\right)\right] .
$$

Remark 3.1. Contrary to the above section, we do not fix here the path of the control $\nu$ on $[0, t]$. This is due to the fact that $X_{t, x}^{\nu}$ depends on $\nu$ only on $(t, T]$, since the probability of having a jump a time $t$ is equal to 0 . Moreover, we can always reduce in the above definition to controls $\nu$ in $\mathcal{U}$ that are independent of $\mathcal{F}_{t}$, see Remark 5.2 in [7].

Then, it follows from standard arguments, see [12] or [19] and the recent paper [7], that the lower-semicontinuous envelope $v_{*}$ and the upper-semicontinuous envelope $v^{*}$ of $v$ defined as

$v_{*}(t, x):=\liminf _{\left(t^{\prime}, x^{\prime}\right) \rightarrow(t, x), t^{\prime}<T} v\left(t^{\prime}, x^{\prime}\right)$ and $v^{*}(t, x):=\limsup _{\left(t^{\prime}, x^{\prime}\right) \rightarrow(t, x), t^{\prime}<T} v\left(t^{\prime}, x^{\prime}\right),(t, x) \in[0, T] \times \mathbb{R}^{d}$ satisfy:

Theorem 3.2. Assume that $v$ is locally bounded. Then, $v_{*}$ is a viscosity supersolution of

$$
\begin{array}{rll}
-\partial_{t} \varphi+H^{*}\left(\cdot, D \varphi, D^{2} \varphi, \varphi\right)=0 & \text { on } & {[0, T) \times \mathbb{R}^{d}} \\
(\varphi-g) \mathbf{1}_{H^{*}\left(\cdot, D \varphi, D^{2} \varphi, \varphi\right)<\infty}(T, \cdot)=0 & \text { on } & \mathbb{R}^{d},
\end{array}
$$

where $H^{*}$ is the upper-semicontinuous envelope of the lower-semicontinuous map

$$
\begin{aligned}
H: & {[0, T] \times \mathbb{R}^{d} \times \mathbb{R}^{d} \times \mathbb{M}^{d} \times C^{0}\left([0, T] \times \mathbb{R}^{d}\right) \rightarrow \mathbb{R} } \\
& (t, x, q, A, f) \mapsto \sup _{u \in K}\left(-I[f](t, x, u)-\mu(x, u)^{\top} q-\frac{1}{2} \operatorname{Tr}\left[\sigma \sigma^{\top}(x, u) A\right]\right),
\end{aligned}
$$

with

$$
I[f](t, x, u):=\int_{E}(f(t, x+\beta(x, u, e))-f(t, x)) m(d e) .
$$


Moreover, $v^{*}$ is a viscosity subsolution of

$$
\begin{array}{rll}
-\partial_{t} \varphi+H\left(\cdot, D \varphi, D^{2} \varphi, \varphi\right)=0 & \text { on } & {[0, T) \times \mathbb{R}^{d}} \\
(\varphi-g)(T, \cdot)=0 & \text { on } & \mathbb{R}^{d} .
\end{array}
$$

On the other hand, it follows from (3.2) and the linear growth condition on $g$ that $g\left(X_{t, x}^{\nu}(T)\right) \in$ $L^{2}\left(\Omega, \mathcal{F}_{T}, \mathbb{P}\right)$ for all $\nu \in \mathcal{U}$. The martingale representation theorem then implies that $(2.2)$ holds for the family of martingales $\mathcal{M}$ defined as

$$
\mathcal{M}:=\mathbb{R}+\left\{\int_{0}^{\cdot} \alpha_{s}^{\top} d W_{s}+\int_{0}^{\cdot} \int_{E} \gamma_{s}(e) \tilde{N}(d e, d s),(\alpha, \gamma) \in \mathcal{A} \times \Gamma\right\}
$$

where $\mathcal{A}$ denotes the set of square integrable $\mathbb{R}^{d}$-valued predictable processes, and $\Gamma$ is the set of $\mathcal{P} \otimes \mathcal{E}$ measurable maps $\gamma: \Omega \times[0, T] \times E \rightarrow \mathbb{R}$ such that

$$
\mathbb{E}\left[\int_{0}^{T} \int_{E}\left|\gamma_{s}(e)\right|^{2} m(d e) d s\right]<\infty,
$$

with $\mathcal{P}$ defined as the $\sigma$-algebra of $\mathbb{F}$-predictable subsets of $\Omega \times[0, T]$.

Hence, we deduce from Lemma 2.1 that

$$
v(t, x)=\inf \left\{p \in \mathbb{R}: \exists(\nu, \alpha, \gamma) \in \mathcal{U} \times \mathcal{A} \times \Gamma \text { s.t. } P_{t, p}^{\alpha, \gamma}(T) \geq g\left(X_{t, x}^{\nu}(T)\right)\right\},
$$

where

$$
P_{t, p}^{\alpha, \gamma}:=p+\int_{t}^{\cdot} \alpha_{s}^{\top} d W_{s}+\int_{t}^{\cdot} \int_{E} \gamma_{s}(e) \tilde{N}(d e, d s) .
$$

We therefore retrieve a stochastic target problem in the form studied in [14].

If $v$ is locally bounded, we can then apply the main result of [14] (see [5] for the case of Brownian diffusion models) to deduce that $v_{*}$ is a viscosity supersolution of

$$
\begin{array}{rll}
-\partial_{t} \varphi+F_{0,0}^{*}\left(\cdot, D \varphi, D^{2} \varphi, \varphi\right)=0 & \text { on } & {[0, T) \times \mathbb{R}^{d}} \\
(\varphi-g) \mathbf{1}_{F_{0,0}^{*}\left(\cdot, D \varphi, D^{2} \varphi, \varphi\right)<\infty}(T, \cdot)=0 & \text { on } & \mathbb{R}^{d}
\end{array}
$$

where $F^{*}$ is the upper-semicontinuous envelope of the map $F$ defined for $(\varepsilon, \eta, t, x, q, A, f) \in$ $[0,1] \times[-1,1] \times[0, T] \times \mathbb{R}^{d} \times \mathbb{R}^{d} \times \mathbb{M}^{d} \times C^{0}\left([0, T] \times \mathbb{R}^{d}\right)$ by

$$
F_{\varepsilon, \eta}(t, x, q, A, f):=\sup _{(u, a, b) \in \mathcal{N}_{\varepsilon, \eta}(t, x, q, f)}\left(-\int_{E} b(e) m(d e)-\mu(x, u)^{\top} q-\frac{1}{2} \operatorname{Tr}\left[\sigma \sigma^{\top}(x, u) A\right]\right),
$$

with $\mathcal{N}_{\varepsilon, \eta}(t, x, q, f)$ defined as the set of elements $(u, a, b) \in K \times \mathbb{R}^{d} \times L^{2}(E, \mathcal{E}, m)$ such that

$$
\left|a-\sigma(x, u)^{\top} q\right| \leq \varepsilon \text { and } b(e)-f(t, x+\beta(x, u, e))+f(t, x) \geq \eta \text { for } m \text {-a.e } e \in E .
$$

Similarly, Theorem 2.1 in [14] states that $v^{*}$ is a viscosity subsolution of

$$
\begin{aligned}
& -\partial_{t} \varphi+F_{0,0 *}\left(\cdot, D \varphi, D^{2} \varphi, \varphi\right)=0 \quad \text { on } \quad[0, T) \times \mathbb{R}^{d} \\
& (\varphi-g)(T, \cdot)=0 \quad \text { on } \quad \mathbb{R}^{d},
\end{aligned}
$$

where $F_{*}$ is the lower-semicontinuous envelope of $F$.

To retrieve the result of Theorem 3.2, it suffices to show the following. 
Proposition 3.3. $F_{0,0}^{*} \leq H^{*}$ and $F_{0,0 *} \geq H$.

Proof. First note that $u \in K$ implies that $\left(u, \sigma(x, u)^{\top} q, f(t, x+\beta(x, u, \cdot))-f(t, x)+\eta\right) \in$

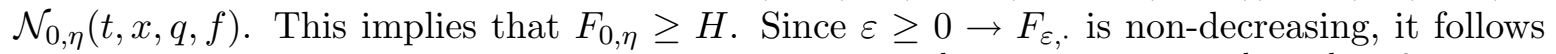
that $F_{0,0 *} \geq H$. On the other hand, fix $(t, x) \in[0, T) \times \mathbb{R}^{d}$ and $(q, A, f) \in \mathbb{R}^{d} \times \mathbb{M}^{d} \times C^{0}([0, T] \times$ $\left.\mathbb{R}^{d}\right)$, and consider a sequence $\left(\varepsilon_{n}, \eta_{n}, t_{n}, x_{n}, q_{n}, A_{n}, f_{n}\right)_{n}$ that converges to $(0,0, t, x, q, A, f)$ such that

$$
\lim _{n \rightarrow \infty} F_{\varepsilon_{n}, \eta_{n}}\left(t_{n}, x_{n}, q_{n}, A_{n}, f_{n}\right)=F_{0,0}^{*}(t, x, q, A, f) .
$$

Then, by definition of $\mathcal{N}_{\varepsilon_{n}, \eta_{n}}\left(t_{n}, x_{n}, q_{n}, f_{n}\right)$, we have

$$
\begin{aligned}
& F_{0,0}^{*}(t, x, q, A, f) \\
& =\lim _{n \rightarrow \infty} F_{\varepsilon_{n}, \eta_{n}}\left(t_{n}, x_{n}, q_{n}, A_{n}, f_{n}\right) \\
& \leq \lim _{n \rightarrow \infty}\left(\left|\eta_{n}\right| m(E)+\sup _{u \in K}\left(-I\left[f_{n}\right]\left(t_{n}, x_{n}, u\right)-\mu\left(x_{n}, u\right)^{\top} q_{n}-\frac{1}{2} \operatorname{Tr}\left[\sigma \sigma^{\top}\left(x_{n}, u\right) A_{n}\right]\right)\right) \\
& \leq H^{*}(t, x, q, A, f) .
\end{aligned}
$$

Remark 3.4. It is clear that the same ideas apply to various classes of optimal control problems: singular control, optimal stopping, impulse control, problem involving state constraints, etc. We refer to [3] and [8] for the study of stochastic target problems with controls including bounded variation processes or stopping times. The case of state constraints is discussed in [8], see also [4].

\section{References}

[1] B. Bouchard. Stochastic Target with Mixed diffusion processes. Stochastic Processes and their Applications, 101, 273-302, 2002.

[2] B. Bouchard. A stochastic target formulation for optimal switching problems in finite horizon. Stochastics, 81(2), 171-197, 2009.

[3] B. Bouchard and N. M. Dang. Generalized stochastic target problems for pricing and partial hedging under loss constraints - Application in optimal book liquidation. Preprint Ceremade, University Paris-Dauphine, 2010.

[4] B. Bouchard, R. Elie, and C. Imbert. Optimal Control under Stochastic Target Constraints. SIAM Journal on Control and Optimization, 48(5), 3501-3531, 2010.

[5] B. Bouchard, R. Elie, and N. Touzi. Stochastic target problems with controlled loss. SIAM Journal on Control and Optimization, 48(5), 3123-3150, 2009.

[6] B. Bouchard and N. Touzi. Explicit solution of the multivariate super-replication problem under transaction costs. Annals of Applied Probability, 10, 685-708, 2000.

[7] B. Bouchard and N. Touzi. Weak Dynamic Programming Principle for Viscosity Solutions. Preprint Ceremade, University Paris-Dauphine, 2009. 
[8] B. Bouchard and T. N. Vu. The American version of the geometric dynamic programming principle, Application to the pricing of american options under constraints. Applied Mathematics and Optimization, 61(2), 235-265, 2010.

[9] B. Bouchard and T. N. Vu. A PDE formulation for P\&L Matching Problems. In preparation.

[10] P. Brémaud. Point Processes and Queues - Martingale Dynamics. Springer-Verlag, NewYork, 1981.

[11] N. El Karoui. Les aspects probabilistes du contrôle stochastique. Ecole d'Eté de Probabilités de Saint Flour IX, Lecture Notes in Mathematics 876, Springer Verlag, 1979.

[12] W. H. Fleming and H. M. Soner. Controlled Markov Processes and Viscosity Solutions. Second Edition, Springer, 2006.

[13] I. Karatzas and S.E. Shreve. Methods of Mathematical Finance. Springer Verlag, 1998.

[14] L. Moreau. Stochastic Target Problems with Controlled Loss in jump diffusion models. Preprint Ceremade, University Paris-Dauphine, 2010.

[15] H. M. Soner and N. Touzi. Super-replication under Gamma constraint. SIAM Journal on Control and Optimization, 39, 73-96, 2000.

[16] H. M. Soner and N. Touzi. Stochastic target problems, dynamic programming and viscosity solutions. SIAM Journal on Control and Optimization, 41, 404-424, 2002.

[17] H. M. Soner and N. Touzi. Dynamic programming for stochastic target problems and geometric flows. Journal of the European Mathematical Society, 4, 201-236, 2002.

[18] N. Touzi. Direct characterization of the value of super-replication under stochastic volatility and portfolio constraints. Stochastic Processes and their Applications, 88, 305-328, 2000 .

[19] J. Yong and X. Zhou. Stochastic Controls: Hamiltonian Systems and HJB Equations. Springer, New York, 1999. 